\title{
A fourth generation, anomalous like-sign dimuon charge asymmetry and the LHC
}

\author{
Debajyoti Choudhury ${ }^{a}$ and Dilip Kumar Ghosh ${ }^{b}$ \\ a Department of Physics $\mathscr{B}$ Astrophysics, \\ University of Delhi, Delhi - 110 00\%, India \\ ${ }^{b}$ Department of Theoretical Physics, \\ Indian Association for the Cultivation of Science, \\ $2 A$ \& B Raja S.C. Mullick Road, Kolkata 700 032, India
}

\begin{abstract}
A fourth chiral generation, with $m_{t^{\prime}}$ in the range $\sim(300-500) \mathrm{GeV}$ and a moderate value of the $\mathrm{CP}$-violating phase can explain the anomalous like-sign dimuon charge asymmetry observed recently by the D0 collaboration. The required parameters are found to be consistent with constraints from other $B$ and $K$ decays. The presence of such quarks, apart from being detectable in the early stages of the LHC, would also have important consequences in the electroweak symmetry breaking sector.
\end{abstract}

\section{Introduction}

Charge-parity $(\mathrm{CP})$ violation is one of the key ingredients for the dominance of matter over anti-matter in the present day universe. The presence of a complex phase in the CabbiboKobayashi-Maskwaw (CKM) matrix [1,2] leads to CP violation in the Standard Model(SM). Since the first observation of the CP violation in the Kaon system [3], several measurements have been performed to observe the same in $B$ and $D$ mesons and the experimental findings have, so far, been largely consistent with the prediction of the SM. However, the SM neither admits sufficient $\mathrm{CP}$ violation nor is the phase transition strong enough to explain the observed magnitude of the baryon asymmetry of the universe. This has led to a sustained effort over decades to look for an evidence of CP-violation going beyond what the SM predicts. Indeed, during the past few years some experimental data collected at Tevatron and B-factories in the heavy flavour-sector indicates mild conflict with the CKM picture of $\mathrm{CP}$ violation within the SM [4 6]. Several new scenarios have been proposed to explain these 
anomalies [7-12]. Much interest has been garnered by the recent observation [13, by the D0 Collaboration, of an anomalous like-sign dimuon charge asymmetry. They find an excess of negatively-charged dimuon pair over positively charged ones, namely $N^{--} \equiv N\left(\mu^{-} \mu^{-}\right)>$ $N^{++}$. Reconstructing the events, and subtracting the backgrounds, they conclude that the only possible explanation is offered by the conjecture that a $b+\bar{b}$, created in a hard process, hadronize into a pair of neutral $B$-mesons (say, $B_{s}+\bar{B}_{s}$ ) each of which suffers a semileptonic $\left[B(\bar{B}) \rightarrow \mu^{-}\left(\mu^{+}\right)+X\right]$ decay. The oscillation of one into the other allows for a 'wrong-sign' decay leading to like-sign dimuons. The asymmetry, then, would be a consequence of unequal probabilities of $B_{s} \rightarrow \bar{B}_{s}$ and $\bar{B}_{s} \rightarrow B_{s}$ oscillations, a manifestation of CP-noninvariance. Thus, the D0 measurement [13], using $6.1 \mathrm{fb}^{-1}$ of data, of

$$
A_{s l}^{b} \equiv \frac{N_{b}^{++}-N_{b}^{--}}{N_{b}^{++}+N_{b}^{--}}=-(9.57 \pm 2.51 \pm 1.46) \times 10^{-3}
$$

would amount to a $3.2 \sigma$ deviation away from the SM prediction of $-0.2 \times 10^{-3}$. On the other hand, the CDF Collaboration [14], using $1.6 \mathrm{fb}^{-1}$ of data has also measured $A_{s l}^{b}$ with a positive central value, namely $A_{s l}^{b}=(8.0 \pm 9.0 \pm 6.8) \times 10^{-3}$. Because of the large errors in the CDF measurements, it is still compatible with the D0 one within $1.5 \sigma$ level. Combining the two, one obtains

$$
A_{s l}^{b} \approx-(8.5 \pm 2.8) \times 10^{-3},
$$

which still is $3 \sigma$ away from the SM value.

It should be noted at this stage that the like-sign charge asymmetry $A_{s l}^{b}$ measured by D0 Collaboration [13] is related to the semileptonic decay asymmetries $a_{s l}^{d}$ and $a_{s l}^{s}$ in the $B_{d}$ and $B_{s}$ mesons, respectively, through

$$
A_{s l}^{b}=(0.506 \pm 0.043) a_{s l}^{d}+(0.494 \pm 0.043) a_{s l}^{s},
$$

where

$$
a_{s l}^{q} \equiv \frac{\Gamma\left(\bar{B}_{q} \rightarrow \mu^{+} X\right)-\Gamma\left(B_{q} \rightarrow \mu^{-}+X\right)}{\Gamma\left(\bar{B}_{q} \rightarrow \mu^{+} X\right)+\Gamma\left(B_{q} \rightarrow \mu^{-}+X\right)} \quad(q=d, s) .
$$

The D0 Collaboration has also directly measured $a_{s l}^{s}$, albeit with much large errors [15]

$$
a_{s l}^{s}=-\left(1.7 \pm 9.1_{-1.5}^{+1.4}\right) \times 10^{-3},
$$


whereas the current direct bound on $a_{s l}^{d}$ reads [16]

$$
a_{s l}^{d}=(4.7 \pm 4.6) \times 10^{-3} .
$$

At this stage, it should be realized that, within the SM, $a_{s l}^{d}$ would contribute negligibly to $A_{s l}^{b}$. And since the new physics (NP) scenario that we would be considering entails almost a vanishingly small contribution to this quantity, it is meaningful to assume a SM value for the same. Thus, combining all the data, we have [17]

$$
a_{s l}^{s} \approx-(12.7 \pm 5.0) \times 10^{-3}
$$

This result is still $2.5 \sigma$ away from the SM value [5], namely,

$$
a_{s l}^{s} \approx 0.02 \times 10^{-3}
$$

While a discrepancy of this magnitude cannot be considered a definitive discovery of NP effects, it certainly adds to the existing list of deviations from the SM expectations seen in the $b$-sector. It, thus, becomes interesting to consider well-motivated NP scenarios that might lead to an coherent explanation of such anomalies while leading itself to experimental verification in other independent channels [17 23]. Perhaps the simplest such extension is the postulation of a fourth family of quarks that mix with the three known families, thereby altering the structure of meson-mixings, CP violation, flavour-changing neutral currents (FCNC) etc. [24 36]. A striking consequence of such a fourth family would be the introduction of additional phases in the analogue of the CKM matrix, or in other words, the existence of new Jarlskog invariants [37]. As some of these are no longer suppressed by the first generation quark masses, there is an enormous enhancement in the effective magnitude of $\mathrm{CP}$ violation available to the mechanism of baryogenesis [38].

Although the addition of vector-representations of quarks is, in some sense, minimal and also suffices to explain the long-standing anomaly in the forward-backward asymmetry in $b$ pair production at LEP/SLC [39], the introduction of such representations typically results in tree-level FCNCs [40]. We, rather, choose to work with the more canonical scenario, namely a chiral fourth generation $\left(t^{\prime}, b^{\prime}\right)$ quark model (SM4).

The CDF collaboration has looked for the existence of such quarks and quote bounds of $m_{t^{\prime}}>335 \mathrm{GeV}$ [41] and $m_{b^{\prime}}>338 \mathrm{GeV}$ [42] respectively. However, as both these analyses assume a 100\% decay branching fraction into particular modes, the model-independent 
bounds would be relaxed somewhat and heavy quarks of $m_{t^{\prime}, b^{\prime}} \gtrsim 300 \mathrm{GeV}$ are still quite consistent. On the other hand, the introduction of chiral fermions can cause deviations in electroweak precision test variables (in particular, the custodial symmetry parameter $\rho$ ) from their SM values, and this severely constrains the allowed mass splitting between the quarks to $\left|m_{t^{\prime}}-m_{b^{\prime}}\right| \lesssim 70 \mathrm{GeV}$ [43 49$]$.

The rest of this article is organized as follows. In Section 2, we discuss the like-sign dimuon charge asymmetry from $B_{s}-\bar{B}_{s}$ mixing in the SM4. We also show the constraints on the parameters space of SM4 obtained from $b \rightarrow s \gamma$ process. In Section 3, we discuss the possible mechanisms for a direct search of heavy $t^{\prime} / b^{\prime}$ quarks at the LHC. Finally, in Section 4 , we summarize our findings.

\section{$2 B_{s}-\bar{B}_{s}$ mixing}

The lighter $(L)$ and the heavier $(H)$ mass eigenstates of the $B_{s}$ system are split with sizeable differences for both mass $\left(\Delta M_{s} \equiv M_{s H}-M_{s L}\right)$ and decay widths $\left(\Delta \Gamma_{s} \equiv \Gamma_{s L}-\Gamma_{s H}\right)$. Within the SM, $B_{s}-\bar{B}_{s}$ mixing is dominated mainly by the box diagrams with top quarks and $W$ boson circulating in the loop. With the introduction of a fourth family, and allowing for it to mix with the known three, the CKM matrix is now extended to a $4 \times 4$ unitary one. As a consequence, additional diagrams with a $t^{\prime}$ replacing one or both of the $t$-quarks in the loop now contribute. The expression for the mass difference is now altered to [29]

$$
\Delta M_{s}=2\left|M_{12}\right|,
$$

where

$$
M_{12}=\frac{G_{F}^{2} m_{W}^{2}}{12 \pi^{2}} m_{B_{s}} B_{b s} f_{B_{s}}^{2}\left\{\eta_{t} \lambda_{t}^{2} S_{0}\left(x_{t}\right)+\eta_{t^{\prime}} \lambda_{t^{\prime}}^{2} S_{0}\left(x_{t^{\prime}}\right)+2 \tilde{\eta}_{t^{\prime}} \lambda_{t} \lambda_{t^{\prime}} S_{0}\left(x_{t}, x_{t^{\prime}}\right)\right\},
$$

with $x_{t}=m_{t}^{2} / m_{W}^{2}, x_{t^{\prime}}=m_{t^{\prime}}^{2} / M_{W}^{2}$ and $\lambda_{t}=V_{t s}^{*} V_{t b}, \lambda_{t^{\prime}}=V_{t^{\prime} s}^{*} V_{t^{\prime} b}$. The loop integrals $S_{0}(x), S_{0}(x, y)$ can be found in Ref. 229. The quantities $\eta_{t}, \eta_{t^{\prime}}$ and $\tilde{\eta}_{t^{\prime}}$ represent the QCD corrections accrued from running the effective operator obtained by integrating out the heavy fields down to the $B$-meson scale. For example, the SM QCD factor $\eta_{t}=0.5765 \pm 0.0065$ [50], while

$$
\eta_{t^{\prime}}=\left[\alpha_{s}\left(m_{t}\right)\right]^{6 / 23}\left[\frac{\alpha_{s}\left(m_{b}^{\prime}\right)}{\alpha_{s}\left(m_{t}\right)}\right]^{6 / 21}\left[\frac{\alpha_{s}\left(m_{t}^{\prime}\right)}{\alpha_{s}\left(m_{b}^{\prime}\right)}\right]^{6 / 19}
$$


with analogous expressions for $\tilde{\eta}_{t^{\prime}}$. Owing to the relatively small running of $\alpha_{s}$ between these scales, numerically, $\eta_{t^{\prime}}, \tilde{\eta}_{t^{\prime}} \approx \eta_{t}$.

The unitarity of the CKM-4 matrix implies $\lambda_{u}+\lambda_{c}+\lambda_{t}+\lambda_{t^{\prime}}=0$. Neglecting $\lambda_{u}$ in comparison with the others (an excellent approximation), we may write

$$
\lambda_{t} \cong-\lambda_{c}-\lambda_{t^{\prime}}
$$

with $\lambda_{c}=V_{c s}^{*} V_{c b}$ being real by convention. For $V_{c b}$, we use the value quoted in Table 1. Parametrizing

$$
\lambda_{t^{\prime}}=r_{t^{\prime}} \exp \left(i \phi_{t^{\prime}}\right)
$$

where $\phi_{t^{\prime}}$ is the new CP violating phase, we can now express all the NP effects essentially in terms of $m_{t^{\prime}}, r_{t^{\prime}}$ and $\phi_{t^{\prime}}$.

\begin{tabular}{lc}
\hline parameter & value \\
\hline$m_{t}\left(m_{t}\right)$ & $(163.5 \pm 1.7)(\mathrm{GeV})$ \\
$\alpha_{s}\left(M_{Z}\right)$ & 0.118 \\
$V_{c b}$ & $(40.8 \pm 0.6) \times 10^{-3}$ \\
$\mathrm{BR}\left(B \rightarrow X_{s} \gamma\right)$ & $(3.55 \pm 0.25) \times 10^{-4}[16]$ \\
$\operatorname{BR}\left(B \rightarrow X_{c} \ell \nu\right)$ & $(10.61 \pm 0.17) \times 10^{-2}[32]$ \\
$f_{b s} \sqrt{B_{b s}}$ & $(0.275 \pm 0.013) \mathrm{GeV}[33]$ \\
\hline
\end{tabular}

Table 1: Values of different input parameters used in this analysis.

Within the SM,

$$
\begin{aligned}
\Delta M_{s}^{\mathrm{SM}} & =(19.82 \pm 1.87) \mathrm{ps}^{-1} \\
\Delta \Gamma_{s}^{\mathrm{SM}} & =(0.096 \pm 0.039) \mathrm{ps}^{-1}
\end{aligned}
$$

where the theoretical uncertainty in $\Delta M_{s}^{\mathrm{SM}}$ arises mainly from the uncertainty in the combination $f_{b s} \sqrt{B_{b s}}$ (see Table 1) of the $B_{s}$ decay constant and the bag parameter. Consequent to the remarkable sensitivity of both the D0 and CDF experiments, the average decay width $\Gamma_{s} \equiv\left(\Gamma_{s L}+\Gamma_{s H}\right) / 2$ and the mass difference $\Delta M_{s}$ have been measured with an accuracy better than $2 \%$ [6, 18, 52, 54] and we now have

$$
\begin{aligned}
\Gamma_{s} & =1.472_{-0.026}^{+0.024} \mathrm{ps}^{-1} \\
\Delta M_{s} & =17.77 \pm 0.10 \pm 0.07 \mathrm{ps}^{-1} .
\end{aligned}
$$


On the other hand, measurements done at CDF and D0 experiments [55, 56] using the technique of angular analysis in $B_{s} \rightarrow J / \psi+\phi(b \rightarrow s c \bar{c})$ decay [57, 58, provide [16]

$$
\begin{aligned}
\Delta \Gamma_{s} & = \pm\left(0.154_{-0.070}^{+0.054}\right) \mathrm{ps}^{-1} \\
\beta_{s}^{J / \psi \phi} & \in\left(0.39_{-0.14}^{+0.18}\right) \cup\left(1.18_{-0.18}^{+0.14}\right),
\end{aligned}
$$

where the last line reflects the two-fold ambiguity $\left(\beta_{s}^{J / \psi \phi} \leftrightarrow \pi-\beta_{s}^{J / \psi \phi}\right)$ in the experimental determination of $\beta_{s}^{J / \psi \phi}$. Note also that only the magnitude of $\Delta \Gamma_{s}$ is determined, and not the sign.

Finally, the semileptonic decay asymmetry $a_{s l}^{s}$ as defined in eq. (44) can be related to the $B_{s}-\bar{B}_{s}$ mixing parameters, viz.,

$$
\tan \phi_{s}=\chi_{s} \equiv a_{s l}^{s} \frac{\Delta M_{s}}{\Delta \Gamma_{s}}
$$

where $\phi_{s}$ contains both the SM phase $(\sim 0)$ and the NP phase $\phi_{t^{\prime}}$.

The above equation is, in some sense, the master formula for our analysis. Of the three quantities defining $\chi_{s}$, the mass difference $\Delta M_{s}$ has been measured very accurately (16). On the other hand, the large error bars in $\Delta \Gamma_{s}$, combined with the ambiguity in its sign, allows for large (and disjoint) swathes of $\chi_{s}$.

Note that there exists some tension between $\Delta \Gamma_{s}^{\mathrm{SM}}$ and $\Delta \Gamma_{s}^{\exp }$, although they cannot yet be deemed to be inconsistent with each other. The difference could be attributable to theoretical uncertainties in the estimation of $\Delta \Gamma_{s}^{\mathrm{SM}}$ from the higher order corrections as well as from the long distance effects, as also to experimental ones. However, if the central values are given any credence, this apparent difference would have profound consequences. The width difference is given by

$$
\Delta \Gamma_{s}=2\left|\Gamma_{12 s}\right| \cos \phi_{s}
$$

where, $\phi_{s} \equiv \operatorname{Arg}\left(-M_{12 s} / \Gamma_{12 s}\right)$ and $\Gamma_{12 s}$ is the absorptive part of the $B_{s}-\bar{B}_{s}$ mixing amplitude. Within the SM [5]

$$
\phi_{s}=0.0041 \pm 0.0007
$$

and, therefore, $\Delta \Gamma_{s}^{\mathrm{SM}} \approx 2\left|\Gamma_{12 s}\right|$. It is worth emphasizing that in SM4, there is no room for additional contribution to $\Gamma_{12 s}$ which implies that $\Delta \Gamma_{s} \leq \Delta \Gamma_{s}^{\mathrm{SM}}$. This is true not only for SM4, but for all theories wherein no additional absorptive parts appear. In other words, 
any new physics scenario that seeks to enhance $a_{s l}^{s}$ without enhancing $\Gamma_{12 s}$ runs the risk of rendering $\left|\sin \phi_{s}\right| \geq 1$. Thus, the only way, for all such theories, to explain the D0 result would be to appeal to the theoretical uncertainties in $\Delta \Gamma_{s}^{S M}$ determination and rather assume that the experimentally measured value reflects the truth accurately. This is the approach we adopt and with this, we may now proceed to find solutions in the three-dimensional parameter space $\left(m_{t^{\prime}}, r_{t^{\prime}}, \phi_{t^{\prime}}\right)$ that are consistent both mathematically and with the data.

Before we do so, though, it is useful to consider an approximate symmetry in the problem. Given the fact that $\phi_{s}^{S M}$ is a small quantity (see eq.20), in the limit of neglecting it, the allowed parameter space would have a $\phi_{t^{\prime}} \leftrightarrow 2 \pi-\phi_{t^{\prime}}$ symmetry. We may, thus, restrict ourselves essentially to $\phi_{t^{\prime}} \in[0, \pi]$. Having done this, for a given combination of $\left(\chi_{s}, m_{t^{\prime}}, r_{t^{\prime}}\right)$, eq,18 turns out to be quartic one in $\cos \phi_{t^{\prime}}$ leading to four solution families. The inclusion of experimental errors would expand each curve into a band, and in Fig 1 we display the $1 \sigma$ allowed regions in the $\phi_{t^{\prime}}-r_{t^{\prime}}$ plane for two representative values of $m_{t^{\prime}}$. Only the (four) strips, each enclosed between a pair of similar curves, are allowed. As expected, the origin (corresponding essentially to a fourth generation decoupled from the known three) lies clearly outside the allowed region.
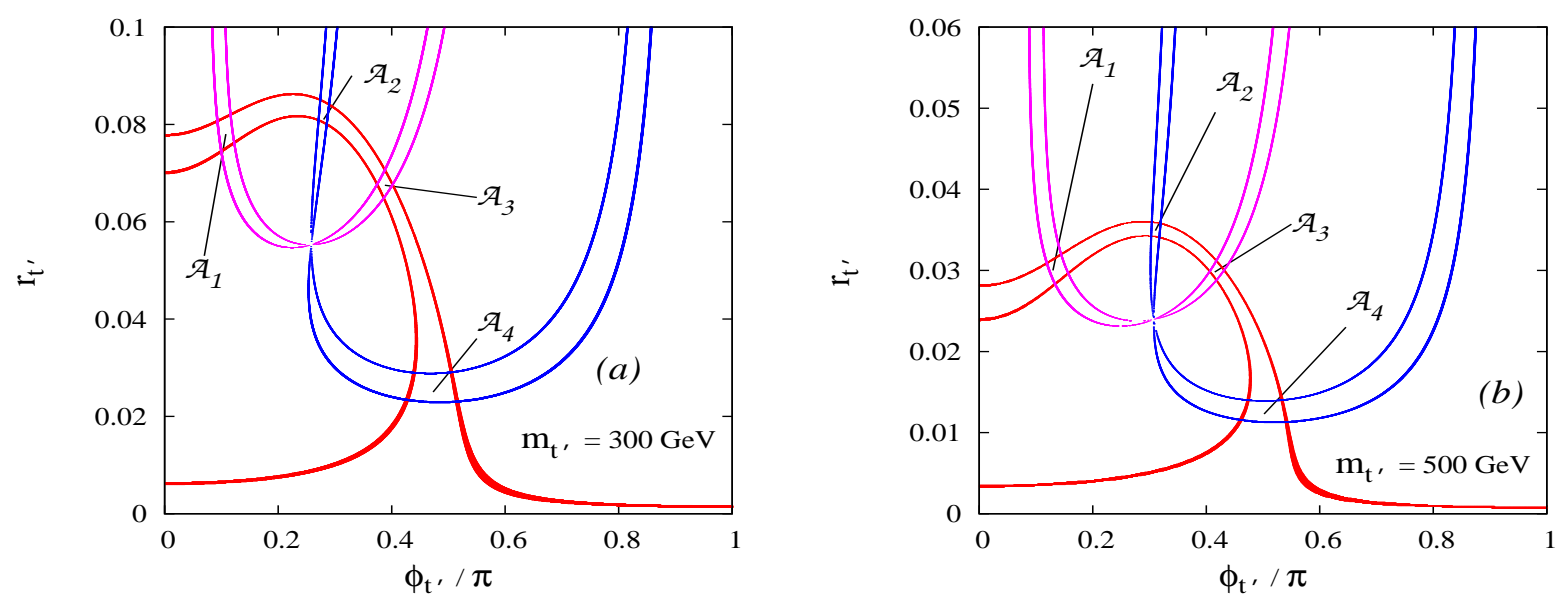

Figure 1: Parts of the parameter space allowed at $1 \sigma$ by eq.18 are given by the four upward convex strips enclosed by red or blue lines. The range allowed by $\Delta M_{s}$ is denoted by the downward convex strip (enclosed by green lines). The four areas $\mathcal{A}_{1,2,3,4}$ are allowed by both observables. The two panels correspond to different values of $m_{t^{\prime}}$.

This parameter space is further constrained by the precise measurement of $\Delta M_{s}$. Solving eq.10 for a given $m_{t^{\prime}}$, we once again get a $1 \sigma$ allowed band in the $\left(\phi_{t^{\prime}}, r_{t^{\prime}}\right)$ plane (see Fig [1). 
Now, of course, the origin is included in the allowed set. The intersection of the two sets, then, gives us the allowed region consistent (within $1 \sigma$ ) with the experimental measurements of both the single charge asymmetry parameter $a_{s l}^{s}$ as well as $\Delta M_{s}$. Note that the said intersection is a union of four disjoint areas of the parameter space, denoted in Fig:1 by $\mathcal{A}_{1,2,3,4}$. It has been argued [36, 48] that the measurement of $Z \rightarrow b \bar{b}$ at LEP and SLC puts very strong constraints on $r_{t^{\prime}} 1$. If we take this at face value, then, of the four disjoint areas, only part of $\mathcal{A}_{4}$ remains. However, apart from the fact that the constraints of Ref. [32] have been obtained with a slightly differing set of inputs and cannot be imposed directly, note that that both our allowed ranges as well as the bounds of Ref. [32] are at $1 \sigma$ and, even at only $1.5 \sigma$, the overlap is quite extensive. Furthermore, the said strong bounds do hinge upon the assumption of no other new physics being of relevance. In view of this, we would advocate that all the four regions be considered seriously and the final outcome be decided by further analyses, both experimental and theoretical.

We now consider possible constraints on the NP parameter space from the measurement of the inclusive $b \rightarrow s \gamma$ transition, namely $\mathrm{B}\left(B \rightarrow X_{s} \gamma\right)$. This one-loop process, in the $\mathrm{SM}$, is dominated by the diagram involving a virtual top quark and $W$ boson. The notso-inconsiderable branching ratio, combined with the theoretical cleanliness make this an ideal theater for testing any new theory bearing on flavour. In the presence of the fourth generation, additional contribution to the $b \rightarrow s \gamma$ amplitude would accrue from the $t^{\prime}$-loop.

The effective Hamiltonian for the $b \rightarrow s \gamma$ process can be written as

$$
\mathcal{H}_{e f f}=\frac{4 G_{F}}{\sqrt{2}} V_{t s}^{*} V_{t b} \sum_{i=1}^{8} C_{i}(\mu) Q_{i}(\mu) .
$$

where the operators $O_{i}(\mu)$ and the Wilson coefficients $C_{i}(\mu)$ may be found in Ref. [59]. The fourth general manifests itself essentially in the modification of the two Wilson coefficients $C_{7}$ and $C_{8}[32]$, namely

$$
C_{7,8}^{\mathrm{tot}}(\mu)=C_{7,8}^{S M}(\mu)+\frac{V_{t^{\prime} s}^{*} V_{t^{\prime} b}}{V_{t s}^{*} V_{t b}} C_{7,8}^{t^{\prime}}(\mu)
$$

where the new contributions $C_{7,8}^{t^{\prime}}$ can be obtained from $C_{7,8}^{S M}$ simply by replacing the top quark mass $m_{t}$ in the latter by $m_{t^{\prime}}$. There exists a large uncertainty in the estimation of the different Wilson coefficients due to the definition of the bottom quark mass. This uncertainty

\footnotetext{
${ }^{1}$ The values of $m_{t^{\prime}}, r_{t^{\prime}}$ and $\phi_{t^{\prime}}$ considered in [36] are consistent with constraint from $K^{+} \rightarrow \pi^{+} \nu \nu$ decay.
} 
can be reduced by considering, instead, the ratio 32

$$
R=\frac{\operatorname{BR}\left(B \rightarrow X_{s} \gamma\right)}{\operatorname{BR}\left(B \rightarrow X_{c} e \bar{\nu}_{e}\right)}
$$

where $\mathrm{BR}\left(B \rightarrow X_{c} e \bar{\nu}_{e}\right)$ represents the semi-leptonic branching ratio of the $B$ meson into charmed states. In the leading logarithmic approximation, the ratio $R$ can be conveniently expressed as

$$
R=\frac{\left|V_{t s}^{*} V_{t b}\right|^{2}}{\left|V_{c b}\right|^{2}} \frac{6 \alpha\left|C_{7}^{\mathrm{tot}}(\mu)\right|^{2}}{\pi f\left(x_{c}\right) \kappa\left(x_{c}\right)}
$$

where, $x_{c} \equiv m_{c} / m_{b}$ and the phase space factor $f\left(x_{c}\right)$ and the QCD correction factor $\kappa\left(x_{c}\right)$ can be found in Refs. [32, 60].
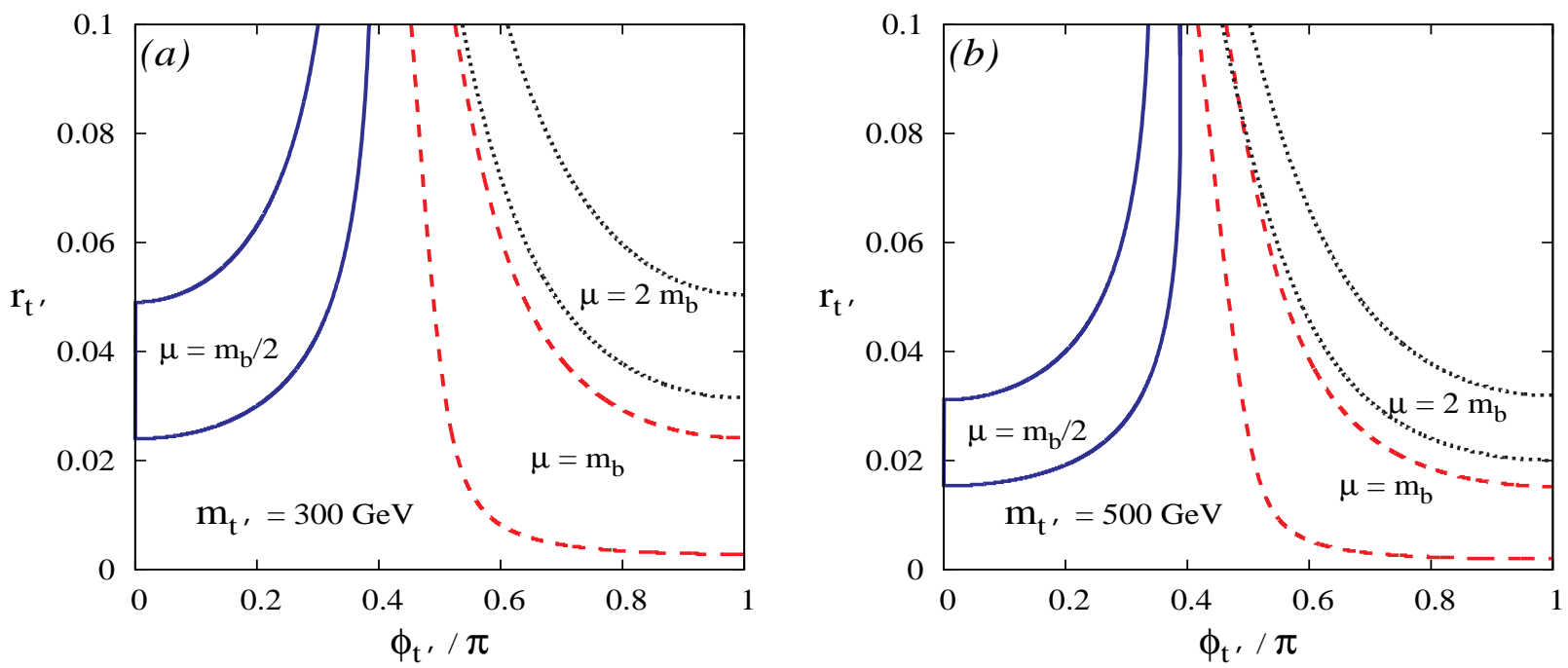

Figure 2: The $1 \sigma$ allowed bands for the ratio $\mathrm{B}\left(B \rightarrow X_{s} \gamma\right) / B\left(B \rightarrow X_{c} e \bar{\nu}_{e}\right)$ in the $\left(\phi_{t^{\prime}}, r_{t^{\prime}}\right)$ plane for two sample choices of $m_{t^{\prime}}$. The dependence on the choice of the scale $\mu$ is also displayed.

In Fig. 2, we display the $1 \sigma$ allowed bands for the ratio $R$ in the $r_{t^{\prime}}-\phi_{t^{\prime}}$ plane for two representative choices of $m_{t^{\prime}}$. Note that there is a very strong dependence on the choice of the scale $\mu$. Given the uncertainty in this choice, it is thus impossible to further constrain the allowed parameter space using this data (as can be easily ascertained by a comparison of Figs. 1 and 2). For a give value of the scale $\mu$, it is obvious that a heavier $t^{\prime}$ implies a smaller $r_{t^{\prime}}$. This is quite analogous to what we also observe in Fig.1 and is easy to understand. Both $\Delta M_{s}$ and $C_{7}^{\text {tot }}$ receive a large positive contribution from the $t^{\prime}$ loop, with the contribution 
growing 2 with $m_{t^{\prime}}$. Thus, to compensate for such large enhancements, $r_{t^{\prime}}$ must become smaller. Indeed, it is the same effect that led Ref. [48] to conclude that electroweak precision tests allow for a smaller $r_{t^{\prime}}$ for larger $m_{t^{\prime}}$.

\section{$3 \quad$ LHC Signals}

It is instructive, at this stage, to consider $t^{\prime}\left(b^{\prime}\right)$ signals at the Tevatron and the LHC 61 67]. Pair production of such quarks is overwhelmingly a pure QCD process (with the $q \bar{q}$ initial state dominating at the Tevatron and the $g g$ state at the LHC) and is analogous to that of top-production, the analytic expressions for which can be found in Ref. [68]. The decay processes, though, depend on the mass splittings and the magnitudes of the CKM-4 matrix elements. Several channels are of interest here. For $m_{t^{\prime}}>m_{b^{\prime}}$, the $t^{\prime}$ dominantly decays into $b^{\prime}+f_{1}+\bar{f}_{2}$ with $f_{i}$ being the SM fermions that are kinematically allowed. The only exception to this would be the case where $t^{\prime}$ and $b^{\prime}$ are closely degenerate and/or the offdiagonal coupling $V_{t^{\prime} b}$ is large. The $b^{\prime}$ decay is more parameter dependent. In general, the dominant decay mode would be $b^{\prime} \rightarrow q+W$ where $q$ is the quark with the dominant offdiagonal coupling with the $b^{\prime}$ (nominally, the top). Again, the exception is provided by the case where $b^{\prime}$ is closely degenerate with the top-quark. In such cases, the loop-mediated decays into $b+Z / H$ could compete with that into $u / c+W$ [69]. On the other hand, for $m_{t^{\prime}}>m_{b^{\prime}}$, the $b^{\prime}$ would like to decay into the $t^{\prime}$ and two soft fermions, unless the $b^{\prime}$ and $t^{\prime}$ are closely degenerate, in which case the $t^{\prime}$ would be replaced by the $t$. As for the $t^{\prime}$, its decay would now be almost overwhelmingly into $b+W$ leading to top-like events [70] but with some differences in the kinematical distributions. Issues such as this have been studied extensively in the context of the Tevatron both experimentally [41,42] and theoretically [71].

Turning to the LHC, we display, in Fig. 3, the cross sections for pair-production of a heavy quark $Q$ (whether $t^{\prime}$ or $b^{\prime}$ ) as a function of its mass. While the calculation for the higher order corrections to $t \bar{t}$ production [72] could be adapted for this case, we desist from doing so. Note that the pair-production could also be accompanied by one or more hard jets. Inclusion of such processes would enhance the cross-section by about 40-50\% almost independent of the heavy-quark mass [70]. Since signal and the background (typically dominated by $t \bar{t}$

\footnotetext{
${ }^{2}$ The decoupling theorem does not hold here, since for large $m_{t^{\prime}}$, it couples to very strongly with longitudinal mode of the $W$ boson.
} 
production) are enhanced in a similar fashion, the inclusion of such events would be expected to increase the statistical significance. This is also helped by the fact that the ISR for $Q \bar{Q}$ production would, typically, tend to be harder than that for $t \bar{t}[73,74]$.
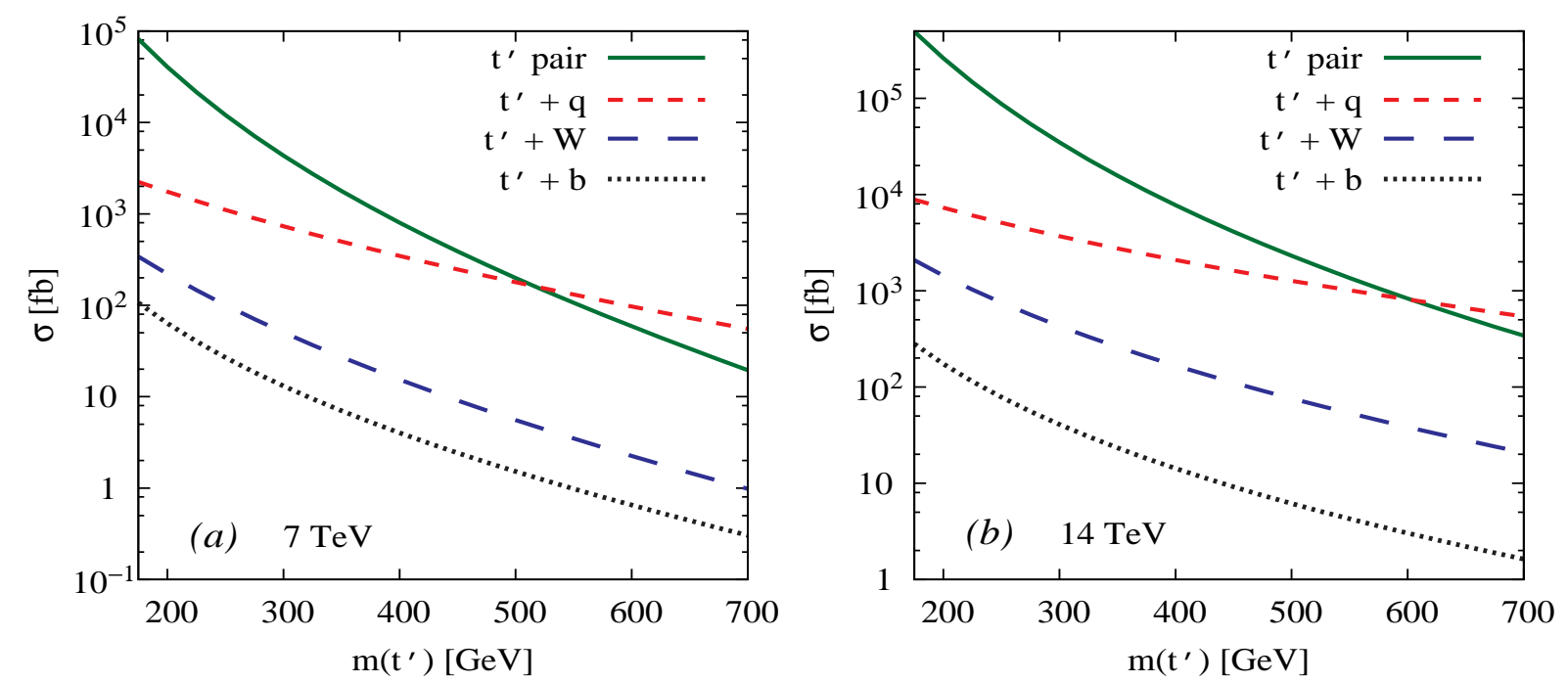

Figure 3: Variation of $t^{\prime}$ production cross sections in different modes as a function of its mass for (a) $\sqrt{s}=7 \mathrm{TeV}$ and (b) $\sqrt{s}=14 \mathrm{TeV}$. In each the solid (green), short-dashed (red), long-dashed (blue) and dotted (black) curves refer to $t^{\prime} \bar{t}^{\prime}, t^{\prime}+$ light $-q, t^{\prime}+W$ and $t^{\prime}+b$ modes respectively. All cross sections are at the leading order and computed with CTEQ6L parton densities. The weak cross sections scale with $\left|V_{t^{\prime} b}\right|^{2}$ and have been computed with $\left|V_{t^{\prime} b}\right|^{2}=0.04$

Perhaps of equal interest is the weak production of these quarks. It is well-known that, at the LHC, single-production of the top-quark is quite comparable to the QCD-driven pairproduction, the smallness of the weak coupling being nearly compensated for by the larger phase-space, enhanced flux and the dynamics. Indeed, such a production mode is of great interest both at the Tevatron and the LHC on account of it being a direct probe of $V_{t b}$.

A similar effect occurs here too. However rather than consider the Cabibbo-unsuppressed process (driven by $V_{t^{\prime} b^{\prime}}$ ), we consider the Cabibbo-suppressed processes driven by $V_{t^{\prime} b}$. Note that a hierarchy similar to that present in the CKM-3, coupled with $\left|V_{t^{\prime} b} V_{t^{\prime} s}^{*}\right| \sim 0.02$ would typically mean $\left|V_{t^{\prime} b}\right| \gtrsim 0.2$. Indeed, a large class of models [75, 76] predict that $\left|V_{t^{\prime} b}\right| \sim\left|V_{u d}\right|$. In Fig [3, we also present the cross sections for three different weak sub-processes, computed with $\left|V_{t^{\prime} b}\right|^{2}=0.04$. It should be understood that these are leading order results and would, 
in general, suffer considerable higher order corrections. Nonetheless, it is instructive to note that, for large $t^{\prime}$-masses, the weak processes are comparable with, or even dominate, the strong-production process. With the final states being quite different, only a detailed analysis of the corresponding background can tell us about the experimental viability of this mode.

It might be argued at this stage that a very heavy fourth generation with considerable mixing with the third family is disfavoured by the electroweak precision tests [48]. This would render irrelevant our observation of single $t^{\prime}$-production being important for large $m_{t^{\prime}}$ values. Note, however, that such observations are contingent upon the 4th family being the only NP source close to the electroweak scale. If this assumption is relaxed, the constraints do change considerably. Direct observation, or the lack of it, would consist the best test.It should be realized, nonetheless, that a very large value of the quark masses would imply a large Yukawa coupling, bordering on nonperturbativity.
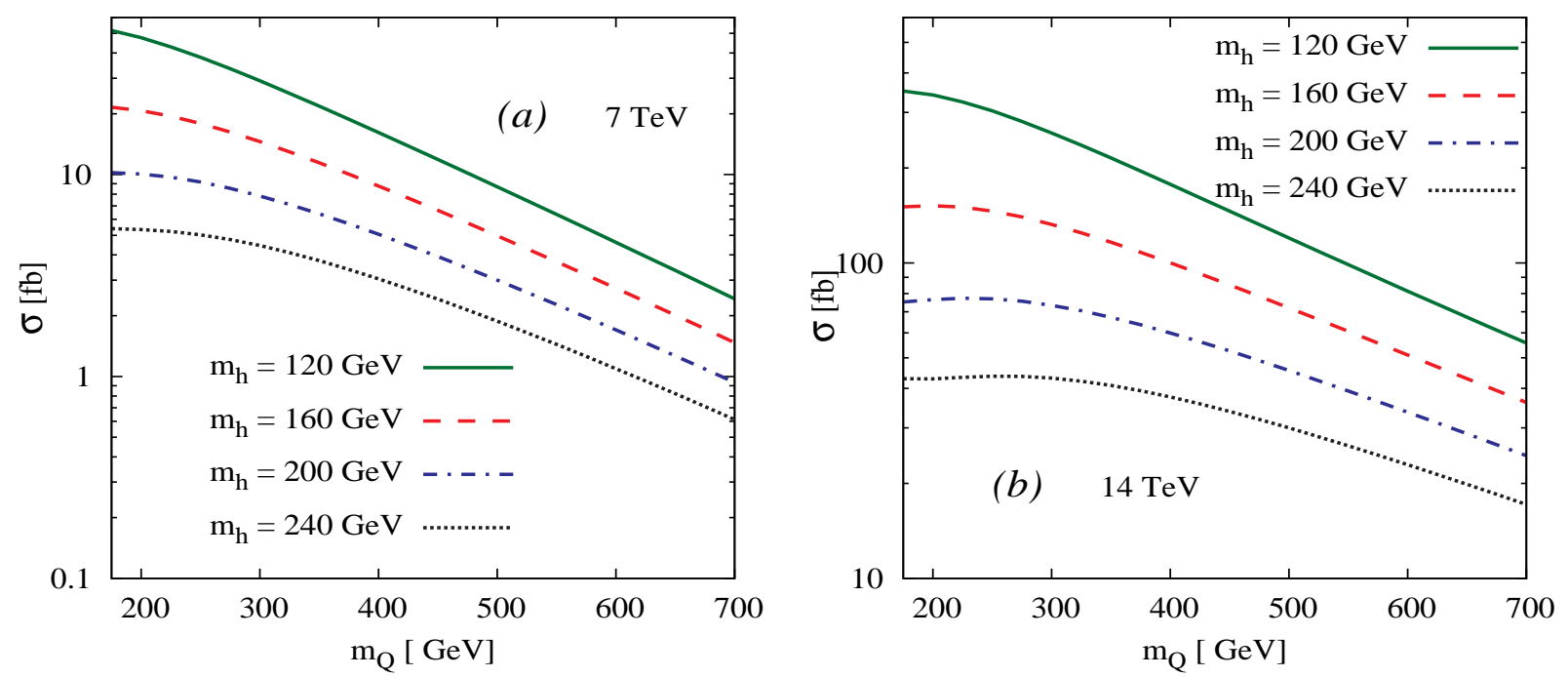

Figure 4: The $Q \bar{Q} h$ production cross section at the $L H C$ as a function of $m_{Q}$ for $(\mathrm{a}) \sqrt{s}=$ $7 \mathrm{TeV}$ and (b) $\sqrt{s}=14 \mathrm{TeV}$. In each the solid (green), short-dashed (red), long-dashed (blue) and dotted (black) curves refer to $m_{h}=120,160,200,240 \mathrm{GeV}$ respectively. All cross sections are at the leading order and computed with CTEQ6L parton densities.

A further consequence of the existence of such heavy quarks, and one almost independent of the magnitude of $V_{t^{\prime} b}$ is the associated production of the Higgs boson. The process $p p \rightarrow t \bar{t} h+X$ has been well-studied in the context of the LHC and both both ATLAS 
[77] and CMS collaborations [78] have run extensive simulations. While initially it seemed that, experimentally, the channel was of marginal relevance, a recent reanalysis [79] argues otherwise and, indeed, advocates its use to even measure the top Yukawa coupling. With the addition of the fourth family, both $p p \rightarrow t^{\prime} \bar{t}^{\prime} h+X$ and $p p \rightarrow b^{\prime} \bar{b}^{\prime} h+X$ become relevant. While the higher masses of the quarks would cause kinematic suppression, they also imply an enhanced Yukawa coupling. Although the former effect does win (see Fig, 4) the suppression in the cross section with $m_{Q}$ is not very steep. Indeed, for $m_{t^{\prime}} \lesssim 500 \mathrm{GeV}$ (the preferred range, as discussed above), the two modes above, together, lead to a sizable increase in the associated Higgs production cross section, rendering it a very interesting mode at the LHC. It should also be realized that the presence of such quarks would enhance the $g g \rightarrow h$ cross section as well [47]. While, for a light Higgs, the experimentally important two-photon decay mode also suffers a change, the last effect is not relevant for $m_{h} \gtrsim 160 \mathrm{GeV}$.

\section{Summary}

To summarise, we have sought to explain the recently claimed evidence for an anomalous asymmetry in like-sign dimuon events by the D0 Collaboration [13] in terms of a possible fourgeneration extension of the Standard Model. While a degenerate fourth-family is protected from both electroweak precision tests as well as tree-level FCNCs, the inclusion of such extra quarks, immediately leads to the possibility of additional quark-mixing, and, hence, to additional sources of CP-violation. Assuming, for simplicity, that the mixing of the 4th generation with the first one is negligibly small, allows one to significantly reduce the number of relevant new parameters, essentially to the mass of the $t^{\prime}$ and $b^{\prime}$ (which need to be relatively degenerate to protect deviations from custodial symmetry), and the magnitude and phase of $\lambda_{t^{\prime}} \equiv V_{t^{\prime} b} V_{t^{\prime} s}$. Confronting such a model with the experimental data, we find that the measured value of the single-lepton decay asymmetry $a_{s l}^{s}$ and the mass difference $\Delta M_{s}$ impose complementary constraints on the parameter space. For a given $m_{t^{\prime}}$, only four narrow regions in the mixing parameter space are allowed.

If no other new physics effects are around the corner, then the LEP and SLC data on $Z \rightarrow b \bar{b}$ would disfavor two of the allowed regions. However, any such new physics effects and/or a shift in the values of the hadronic parameters (such as $B_{b s} f_{b s}^{2}$ ) would change this conclusion to a significant degree. As for $b \rightarrow s \gamma$ transitions, the solutions found herein are 
consistent with the data. Given the large QCD uncertainties and the strong dependence on the scale at which the Wilson coefficients are calculated, the constraints from this arena turns out to be not so crucial.

With a not too-heavy $t^{\prime}$ being favoured by the data, the prospects for detection at the LHC are very good. What is particularly interesting is that the preferred value of $\lambda_{t^{\prime}}$ indicates that single production of $t^{\prime}$-quarks may be an interesting channel to consider. And while such modes do win over the QCD-driven process for very high $m_{t^{\prime}}$ values, it should be noted that such large Yukawa couplings would tend to make the theory non-perturbative relatively early, thereby necessitating the introduction of other new physics, an eventuality also indicated by the electroweak precision tests. And, finally, the presence of such quarks would have a very important bearing on Higgs physics, both in terms of enhancing the glue-glue fusion crosssection as well as through direct associated production (with either of $t^{\prime}$ and $b^{\prime}$ ) rendering it quite important in the LHC context. In other words, if the D0 anomaly stands the test of time and if a fourth generation is the explanation of the same, the experiments at the LHC would soon be in a position to validate it.

\section{Acknowledgments}

DKG acknowledges partial support from the Department of Science and Technology, India under grant SR/S2/HEP-12/2006. The authors would like to thank A. Kundu, A. Lenz for very useful discussions and the Theory Division, CERN for hospitality when this work was initiated.

\section{References}

[1] N. Cabibbo, Phy. Rev. Lett. 10, 531 (1963).

[2] M. Kobayashi and T. Maskawa, Prog. Theor. Phys. 49, 652 (1973).

[3] J. H. Christenson, J. W. Cronin, V. L. Fitch and R. Turlay, Phys. Rev. Lett. 13, 138 (1964).

[4] E. Lunghi and A. Soni, JHEP 0709, 053 (2007); Phys. Lett. B 666, 162 (2008); JHEP 0908, 051 (2009). 
[5] A. Lenz and U. Nierste, JHEP 0706, 072 (2007).

[6] M. Bona et al. [UTfit Collaboration], arXiv:0803.0659 [hep-ph].

[7] K. Agashe, G. Perez and A. Soni, Phys. Lett. 93, 201804 (2004); Phys. Rev. D 71, $016002(2005)$.

[8] M. Blanke, A. J. Buras, B. Duling, S. Gori and T. Weiler, JHEP 0903,001 (2009).

[9] M. Blanke, A. J. Buras, B. Duling, K. Gemmler and S. Gori, JHEP 0903,108 (2009).

[10] S. Casagrande, F. Goertz, U. Haisch, M. Neubert and T. Pfoh, JHEP 0810, 094 (2008).

[11] V. Barger, L. L. Everett, J. Jiang, P. Langacker, T. Liu and C. E. M. Wagner, JHEP 0912,048 (2009).

[12] W. Altmannshofer, A. J. Buras, S. Gori, P. Paradisi and D. M. Straub, Nucl. Phys. B 830,17 (2010).

[13] V. M. Abazov et al. [D0 Collaboration], arXiv:1005.2757[hep-ex].

[14] CDF Collaboration, Measurement of CP asymmetry in semileptonic B decays, Note 9015, October 2007.

[15] V. M. Abazov et al. [D0 Collaboration], Search for CP violation in semileptonic $B_{s}$ decay, arXiv:0904.3907[hep-ex].

[16] E. Barberio et al. [HFAG Collab.], arXiv:0808.1297[hep-ex].

[17] B. A. Dobrescu, P. J. Fox and A. Martin, arXiv:1005.4238[hep-ph].

[18] A. Dighe, A. Kundu and S. Nandi, arXiv:1005.4051 [hep-ph].

[19] C. H. Chen and G. Faisel, arXiv:1005.4582 [hep-ph].

[20] A. J. Buras, M. V. Carlucci, S. Gori, and G. Isidori, arXiv:1005.5310 [hep-ph].

[21] Z. Ligeti, M. Papucci, G. Perez and J. Zupan, arXiv:1006.0432 [hep-ph].

[22] K. S. Babu and J. Julio, arXiv:1006.1092 [hep-ph]. 
[23] N. G. Deshpande, X. G. He and G. Valencia, arXiv:1006.1682 [hep-ph]

[24] W. -S. Hou, R. Willey and A.Soni, Phys. Rev. Lett. 58, 1608 (1987).

[25] W. -S. Hou, A. Soni and H. Steger, Phys. Rev. Lett. 59, 1521 (1987).

[26] W. -S. Hou, A. Soni and H. Steger, Phys. Lett. B 192, 441 (1987).

[27] C. Hamzaoui, A. I. Sanda and A. Soni, Phys. Rev. Lett. 63, 128 (1989).

[28] P. H. Frampton, P. Q. Hung and M. Sher, Phys. Rept. 330,363,(2000).

[29] A. Arhrib and W. S. Hou, Eur. Phys. J. C 27, 555 (2003).

[30] J. Alwall, R. Frederix, J.-M. Gerard, A. Giammanco, M. Herquet, S. Kalinin, E. Kou, V. Lemaitre, F. Maltoni, Eur. Phys. J. C 49,791, (2007).

[31] M. Bobrowski, A. Lenz, J. Riedl and J. Rohrwild, Phys. Rev. D 79 (2009) 113006.

[32] A. Soni, A. K. Alok, A. Giri, R. Mohanta and S. Nandi, arXiv:1002.0595[hep-ph].

[33] A. J. Buras, B. Duling, T. Feldmann, T. Heidsieck, C. Promberger, and S. Recksiegel, arXiv:1002.2126 [hep-ph].

[34] W. S. Hou and C. Y. Ma, arXiv:1004.2186[hep-ph].

[35] O. Eberhardt, A. Lenz and J. Rohrwild, arXiv:1005.3505 [hep-ph].

[36] A. Soni, A. K. Alok, A. Giri, R. Mohanta and S. Nandi, Phys. Lett. B683,302 (2010).

[37] C. Jarlskog, Phys. Rev. Lett. 55, 1039 (1985); W. S. Hou, Y.-Y. Mao and C.-H. Shen, arXiv:1003.4361 [hep-ph]

[38] W. S. Hou, arXiv:0803.1234[hep-ph].

[39] D. Choudhury, T. M. P. Tait and C. E. M. Wagner, Phys. Rev. D 65, 053002 (2002) arXiv:hep-ph/0109097.

[40] D. E. Morrissey and C. E. M. Wagner, Phys. Rev. D 69, 053001 (2004) arXiv:hep-ph/0308001. 
[41] A. Lister [CDF Collaboration], arXiv:0810.3349 [hep-ex].

[42] T. Aaltonen et al. [CDF Collaboration], Phys. Rev. Lett. 104, 091801 (2010) arXiv:0912.1057].

[43] H. -J. He, N. Polonsky and S. Su, Phys. Rev. D 64, 053004 (2001).

[44] J. Erler and P. Langacker, Acta Phys. Polon. B 39, 2595 (2008) arXiv:0807.3023 [hep-ph]]; arXiv:1003.3211 [hep-ph].

[45] V. A. Novikov, L. B. Okun, A. N. Rozanov and M. I. Vysotsky, Phys. Lett. B 529, 111 (2002).

[46] V. A. Novikov, L. B. Okun, A. N. Rozanov and M. I. Vysotsky, JETP Lett. 76, 127 (2002) [Pisma Zh. Eksp. Teor. Fiz. 76, 158 (2002)].

[47] G. D. Kribs, T. Plehn, M. Spannowsky and T. M. P. Tait, Phys. Rev. D 76, 075016 (2007)

[48] M. S. Chanowitz, Phys. Rev. D 79, 113008 (2009).

[49] M. Hashimoto, Phys. Rev. D 81, 075023 (2010).

[50] A.J.Buras, M.Jamin and P.H. Weisz, Nucl. Phys. B 347, 491 (1990).

[51] P. Ball and R. Fleischer, Eur. Phys. J. C 48, 413 (2006).

[52] C. Amsler et al. [Particle Data Group Collaboration], Phys. Lett. B667, 1 (2008), [URL:http://pdg.lbl.gov].

[53] V. M. Abazov et al. [D0 Collaboration], Phys. Rev. Lett. 97, 021802 (2006).

[54] A. Abulencia et al. [CDF Collaboration], Phys. Rev. Lett. 97, 062003 (2006).

[55] T. Aaltonen et al. [CDF Collaboration], Phys. Rev. Lett. 100, 161802 (2008);

V. M. Abazov et al. [DØ Collaboration], Phys. Rev. Lett. 101, 241801 (2008).

[56] CDF/D0 $\Delta \Gamma_{s}, \beta_{s}$ Combination Working Group, CDF/PHYS/BOTTOM/CDFR/9787, D0 Note 5928-CONF, Version 1.1 (July 2009). 
[57] A.S. Dighe, I. Dunietz, H.J. Lipkin and J.L. Rosner, Phys. Lett. B 369,144 (1996).

[58] A.S. Dighe, I. Dunietz and R. Fleischer, Eur. Phys. J. C 6, 647 (1999).

[59] A.J.Buras and M. Munz, Phys. Rev. D 52, 186 (1995).

[60] Y. Nir, Phys. Lett. B 221, 184 (1989).

[61] B. Holdom and Q. S. Yan, arXiv:1004.3031[hep-ph].

[62] B. Holdom, Phys. Lett. B 686, 146 (2010); JHEP 0608, 076 (2006); JHEP 0703, 063 (2007); JHEP 0708, 069 (2007).

[63] E. Arik et al., Phys. Rev. D 58, 117701 (1998).

[64] V. E. Ozcan, S. Sultansoy and G. Unel, arXiv:0802.2621 [hep-ex].

[65] J. A. Aguilar-Saavedra, JHEP 0911, 030 (2009) arXiv:0907.3155 [hep-ph]].

[66] G. Burdman, L. Da Rold, O. Eboli and R. Matheus, Phys. Rev. D 79, 075026 (2009) arXiv:0812.0368 [hep-ph]].

[67] W. Skiba and D. Tucker-Smith, Phys. Rev. D 75, 115010 (2007) arXiv:hep-ph/0701247.

[68] B. L. Combridge, Nucl. Phys. B 151 (1979) 429.

[69] A. Arhrib, W. S. Hou, JHEP 0607, 009 (2006).

[70] D. Choudhury, A. Datta and K. Ghosh, arXiv:0911.4064 [hep-ph].

[71] C. J. Flacco, D. Whiteson, T. M. P. Tait and S. Bar-Shalom, arXiv:1005.1077.

[72] M. Cacciari et al., JHEP 0809 (2008) 127, arXiv:0804.2800 [hep-ph]].

[73] T. Plehn, D. Rainwater and P. Skands, Phys. Lett. B 645, 217 (2007) arXiv:hep-ph/0510144.

[74] J. Alwall, S. de Visscher and F. Maltoni, JHEP 0902, 017 (2009) arXiv:0810.5350 [hep-ph]]. 
[75] C. D. Froggatt and H. B. Nielsen, Nucl. Phys. B 147, 277 (1979).

[76] A. J. Buras, B. Duling, T. Feldmann, T. Heidsieck, C. Promberger and S. Recksiegel, arXiv:1002.2126.

[77] G. Aad et al. [The ATLAS Collaboration], arXiv:0901.0512 [hep-ex].

[78] G. L. Bayatian et al. [CMS Collaboration], J. Phys. G 34, 995 (2007).

[79] T. Plehn, G. P. Salam, M. Spannowsky, Phys. Rev. Lett. 104, 111801 (2010). 